\title{
Redesign of exhaust protection cover for high air flow levelling valve
}

\author{
P.Sripathy ${ }^{*}$, S.Anantharaman ${ }^{*}$, N.Prabhakar ${ }^{* *}$, R.Satheshkumar \\ "Dep't of Mechanical Engineering, College of Engineering,Anna University, Guindy, Chennai, India \\ WABCO India Ltd, Chennai, India
}

\begin{abstract}
Levelling valve is used in automobile air suspension system. It increases or decreases air pressure in suspension bellows whenever load in the vehicle increased or decreased thus, it maintains constant ride height of chassis during varying load and road conditions. Exhaust protection cover of levelling valve is to prevent the entry of foreign substance into the valve during vehicle running condition and during vehicle water wash. The effectiveness of protection cover depends mainly on how it prevents the entry of foreign substance into valve. This paper describes how present 5 piece exhaust protection cover of levelling valve is redesignedas single component by using CFD as a tool to optimize the design.
\end{abstract}

Keywords: CFD, exhaust protection cover, levelling valve,rubber protection cover, rubber boot

\section{INTRODUCTION}

Levelling Valve shown in Fig.1is used in automobile air suspension system. It increases or decreases air pressure in suspension bellows whenever load in the vehicle increased or decreased thus, it maintains constant ride height of chassis during varying load and road conditions. Varying the bellow pressure varies its stiffness. Thus, Levelling valve facilitates load sensitive suspension. Additionally lock valve limits the air pressure to prevent the vehicle platform from being raised above the permissible safe level.

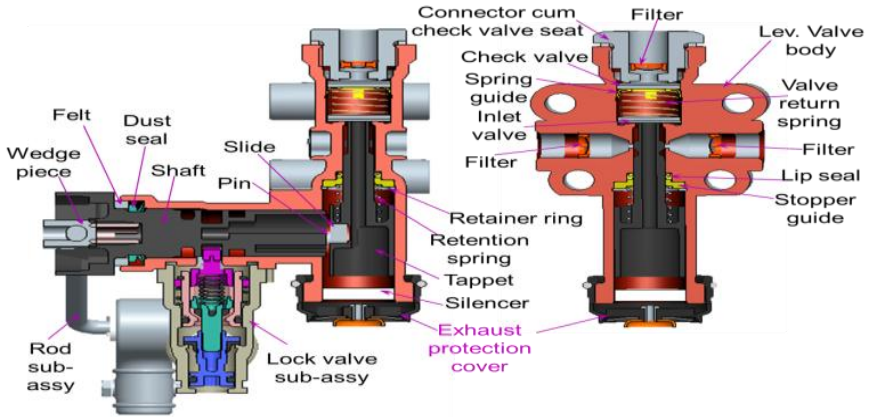

Figure 1 -Levelling valve.

Levelling Valve redesign project is been carried out to reduce the cost of existing levelling valve by providing same function, the main goal for cost reduction is reducing the parts count. In this paper we are discussing how exhaust protection cover is redesigned.

\section{EXHaUst Protection COVER}

An exhaust protection cover of levelling valve is to prevent the entry of foreign substance into valve which will affect the function of valve, especially exhaust protection cover is to prevent the entry of water and mud water into the valve, during vehicle operation at rainy season and during vehicle water wash. Also exhaust protection cover has to withstand the high airflow during the exhaust of valve. The current design of exhaust protection cover, showing its parts used in high flow levelling valve is shown in the Fig.2.

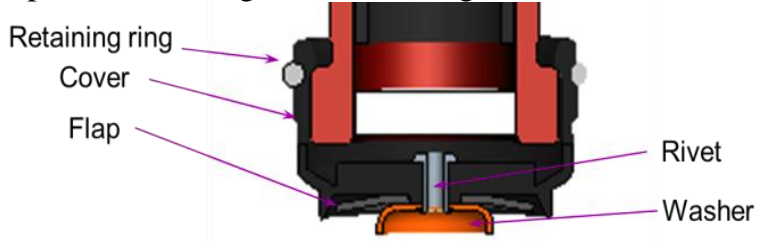

Figure 2-Existing exhaust protection cover.

The existing exhaust protection cover of high flow levelling valve (flow diameter $\varnothing 4 \mathrm{~mm}$ ) is of five parts assembly, which comprises a retaining ring made of spring steel to hold the protection cover in body, a plastic 
protection cover with opening at bottom to allow the flow of air, a rubber flapper which allows only one way flow of air that closes when there is no air flow, preventing the entry of water, a washer and rivet to hold the rubber flap in plastic cover.During the study of various levelling valve it is observed that single piece rubber exhaust protection is used in low flow levelling valve ((flow diameter $\varnothing 3 \mathrm{~mm})$ ). So it is decided to adapt the single piece rubber exhaust protection cover for new high flow levelling valve as shown in Fig.3.

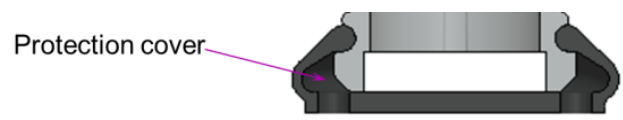

Figure 3-Rubber exhaust protection cover.

Requirements of exhaust protection cover is provided in Table 1, the capability of rubber protection cover for temperature resistance, heat resistance and dust and water protection as per ISO 20653 is already proven in low flow levelling valve. The main task is to check the capability of rubber protection cover for high flow.

\begin{tabular}{|l|c|}
\hline Parameter & Requirement \\
\hline Max. pressure & $10 \mathrm{bar}$ \\
\hline Flow diameter & $4 \mathrm{~mm}$ \\
\hline Temperature range & -40 to $+80{ }^{\circ} \mathrm{C}$ \\
\hline Short term resistance to heat & $+110^{\circ} \mathrm{C}$ for 1 hour \\
\hline Dust and water jet & IP $6 \mathrm{~K} 9 \mathrm{~K}$ as per ISO 20653 \\
\hline
\end{tabular}

Table 1-Requirement for exhaust protection cover.

\section{EXPERIMENTATION}

Experimentation is carried out with existing rubber protection cover for measuring the withstanding capability of standard exhaust protection cover by increasing the pressure from 1 bar to 10 bar gradually. Experimental setup is shown in Fig.4.During experimentation, for 9 bar pressure and measured exhaust flow of $8201 \mathrm{pm}$, where $1 \mathrm{pm}$ - liters per minute, rubber exhaust protection cover is separated from the levelling valve.

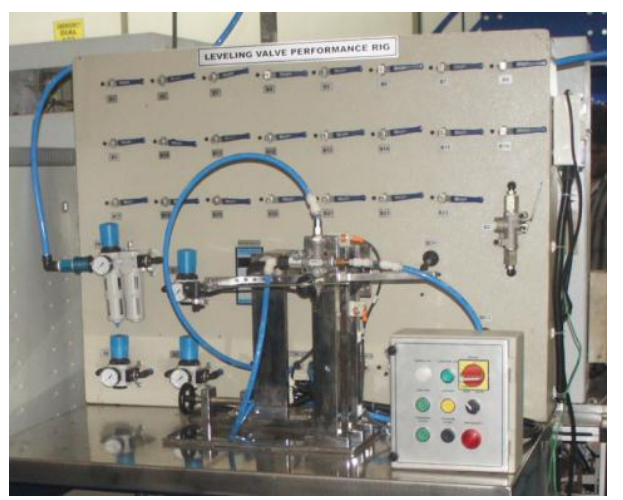

Figure 3-Experimental setup for levelling valve flow measurement.

The rubber protection cover with standing strength can be improved by two ways, one is improving structurally by making rubber exhaust cover more stiffer so that it will not get removed and another way is to reducing the pressure acting on protection cover by optimizing the flow area so that even for high flow with pressure up to 10 bar the protection cover will not get removed, this paper describes how pressure acting on exhaust protection cover is reduced by using CFD as tool.

\section{CFD MODEL DESCRIPTION}

This section illustrates the procedure followed for building a mathematical model, discretization of a computational domain, porous modeling, and CFD solver set up. Autodesk CFD simulation, a design oriented CFD modeling code was selected to investigate and optimize the flow path in the exhaust protection cover, The fluid domain was created by considering the cavity inside the geometry by utilizing the CAD modeling package Pro Engineer. Complete cross section of the leveling valve is shown in the Fig.5 The rubber protection cover is focused for the optimization of the flow path. The other parts were modeled as per the operating requirements of the valves. In order to study the effect of pressurized air flow from valve orifice to exhaust protection cover 
orifice, fluid behavior and flow properties through the circular orifices and elongated circular orifices were discussed theoretically and experimentally validated in [1]. The airflow through the 3D valve passages was considered to be isothermal, compressible and turbulent. Pressure drop at different openings in turbulent flow is discussed in detail in [3]. The Pressure boundary conditions are used to define the fluid pressure at the flow inlet and outlet. The computational model was built such that external structural parts were removed and simplified to reduce the complexity of the problem and the solution time.

An implicit, second order, steady state solver with the SIMPLE algorithm for the pressure velocity coupling is used to solve for the pressure drops through the valve. Convergence of the solution was judged based on residual histories and the convergence criterion was set at 1e-5 for all monitored parameters.
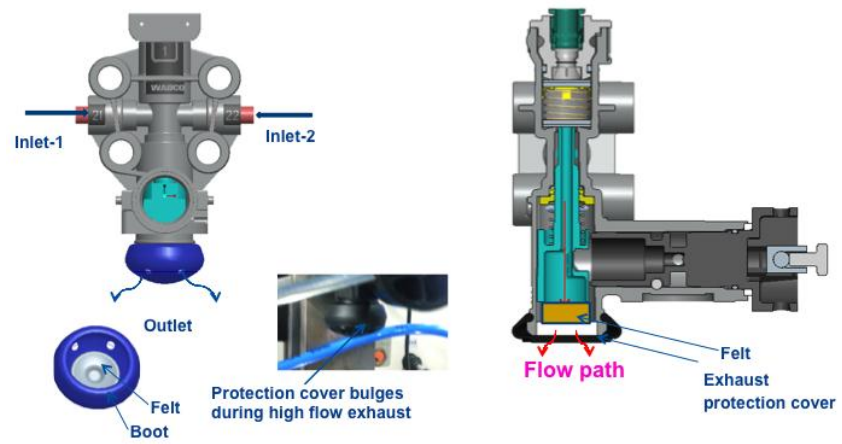

Figure 5-Levelling valve cross section for CFD simulation

Assumptions in modeling are inlet of the valve was extended with the additional caps to obtain fully developed parabolic flow and to reduce entry losses, felt is placed in the exhaust protection cover as like slipping from the body during the test as shown in Fig.5, exhaust protection cover bulging effect is approximately modeled in the modeling software, atmosphere is also modeled to reduce the effect of outlet boundary condition as shown in Fig.6.

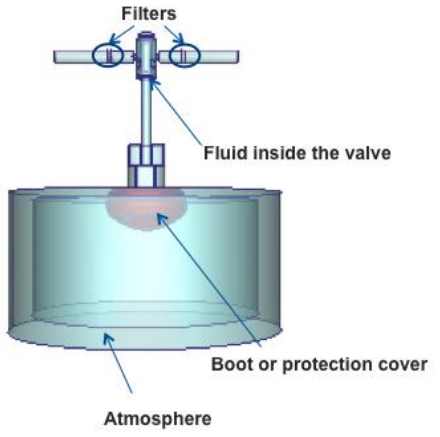

Figure 6-Levelling valve fluid model

\section{CONTINUITY EQUATION}

\section{GOVERNING EQUATIONS}

$$
\frac{\partial \rho}{\partial t}+\frac{\partial \rho u}{\partial x}+\frac{\partial \rho v}{\partial y}+\frac{\partial \rho w}{\partial z}=0
$$

\section{X-MOMENTUM EQUATION}

$$
\begin{aligned}
& \rho \frac{\partial u}{\partial t}+\rho u \frac{\partial u}{\partial x}+\rho v \frac{\partial u}{\partial y}+\rho w \frac{\partial u}{\partial z} \\
& =\rho g_{x}-\frac{\partial p}{\partial x}+\frac{\partial}{\partial x}\left[2 \mu \frac{\partial u}{\partial x}\right]+\frac{\partial}{\partial y}\left[\mu\left(\frac{\partial u}{\partial y}+\frac{\partial v}{\partial x}\right)\right]+\frac{\partial}{\partial z}\left[\mu\left(\frac{\partial u}{\partial z}+\frac{\partial w}{\partial x}\right)\right]+s_{w}+s_{D R}
\end{aligned}
$$

Y-MOMENTUM EQUATION 


$$
\begin{aligned}
& \rho \frac{\partial v}{\partial t}+\rho u \frac{\partial v}{\partial x}+\rho v \frac{\partial v}{\partial y}+\rho w \frac{\partial v}{\partial z} \\
& =\rho g_{y}-\frac{\partial p}{\partial y}+\frac{\partial}{\partial x}\left[\mu\left(\frac{\partial u}{\partial y}+\frac{\partial v}{\partial x}\right)\right]+\frac{\partial}{\partial y}\left[2 \mu \frac{\partial v}{\partial y}\right]+\frac{\partial}{\partial z}\left[\mu\left(\frac{\partial v}{\partial z}+\frac{\partial w}{\partial y}\right)\right]+s_{w}+s_{D R}
\end{aligned}
$$

\section{Z-MOMENTUM EQUATION}

$$
\begin{aligned}
& \rho \frac{\partial W}{\partial t}+\rho u \frac{\partial w}{\partial x}+\rho v \frac{\partial w}{\partial y}+\rho w \frac{\partial w}{\partial z} \\
& \left.=\rho g_{z}-\frac{\partial p}{\partial z}+\frac{\partial}{\partial x}\left[\mu\left(\frac{\partial u}{\partial z}+\frac{\partial w}{\partial x}\right)\right]+\frac{\partial}{\partial y}\left[\mu\left(\frac{\partial v}{\partial z}+\frac{\partial w}{\partial y}\right)\right]+\frac{\partial}{\partial z}\left[2 \mu \frac{\partial w}{\partial z}\right]\right]+s_{w}+s_{D R}
\end{aligned}
$$

The two source terms in the momentum equations are for rotating coordinates and distributed resistances respectively. The distributed resistance $\left(\mathrm{S}_{\mathrm{DR}}\right)$ term can be written in general as

$$
s_{D R}=\frac{\partial p}{\partial x_{i}}
$$

Where,

P-Fluid pressure

t-Time

$\mathrm{u}, \mathrm{v}, \mathrm{w}-$ Velocity in the respective direction $\mathrm{x}, \mathrm{y}, \mathrm{z}$

$\rho$-Fluid Density

$\mu$-Fluid Viscosity

\section{STUDY OF EXISTING DESIGN}

Flow parameters through medium to smaller orifices are discussed in detail by Suresh Gayakwad [2].Theexisting exhaust protection cover which has 6 orifices of diameter $3.5 \mathrm{~mm}$ is studied for various inlet pressures respectively $6,9.10 .5$ bar, where 6 bar is normal operating pressure and 9 bar is experimental failure pressure and 10.5 bar is maximum pressure with 0.5 bar cushion for which the exhaust protection cover has to withstand. The pressure on exhaust protection cover is studied for various inlet pressures, relative comparison of pressure distribution is shown in the protection cover for different inlet pressures in Fig.7. Same pressure contour range is maintained to know the difference in the distribution.

The individual pressure plot is shown in Fig.8, for different inlet pressures. For 6 bar inlet pressure the average and maximum pressure distribution on protection cover are 0.34 bar and 0.475 bar respectively, for 9 bar inlet pressure the average and maximum pressure distribution are 0.58 bar and 0.76 bar respectively and for 10.5 bar inlet pressure the average and maximum pressure distribution on are 0.985 bar and 1.12 bar respectively. So the inference is flow area should be modified in such a way that at maximum inlet pressure of 10.5 bar, average pressure distribution on protection cover should be lesser than 0.58 bar and 0.76 bar respectively, which corresponds to 9 bar failure pressure of existing design. Maximum pressure is initiating the pull out and average pressure is aiding the pull out of protection cover.

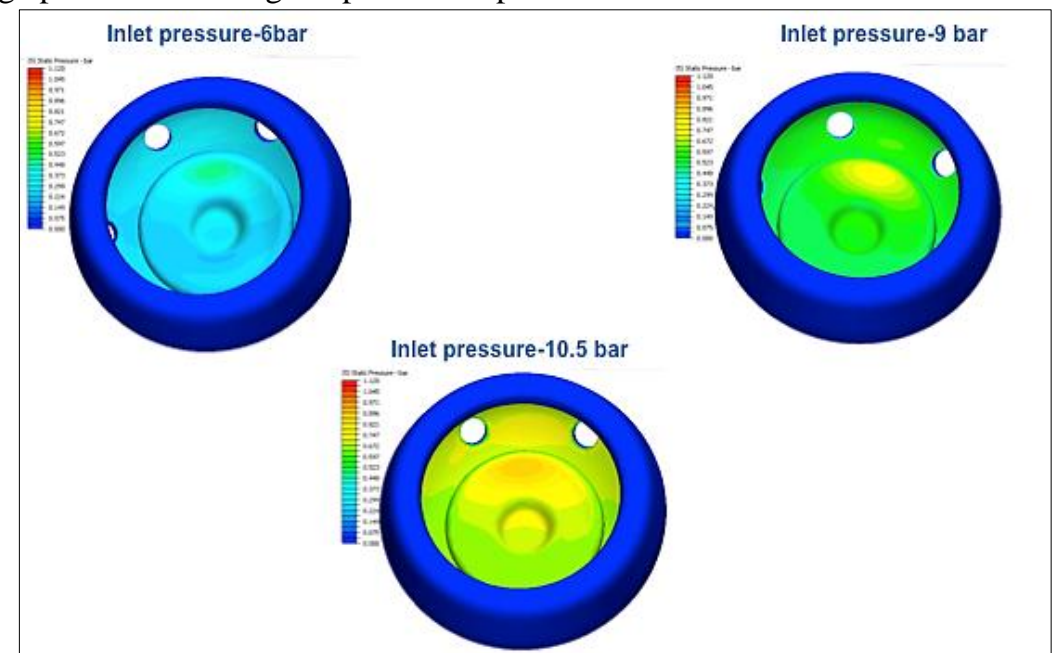

Figure 7 -Pressure distribution for various pressures in same scale 


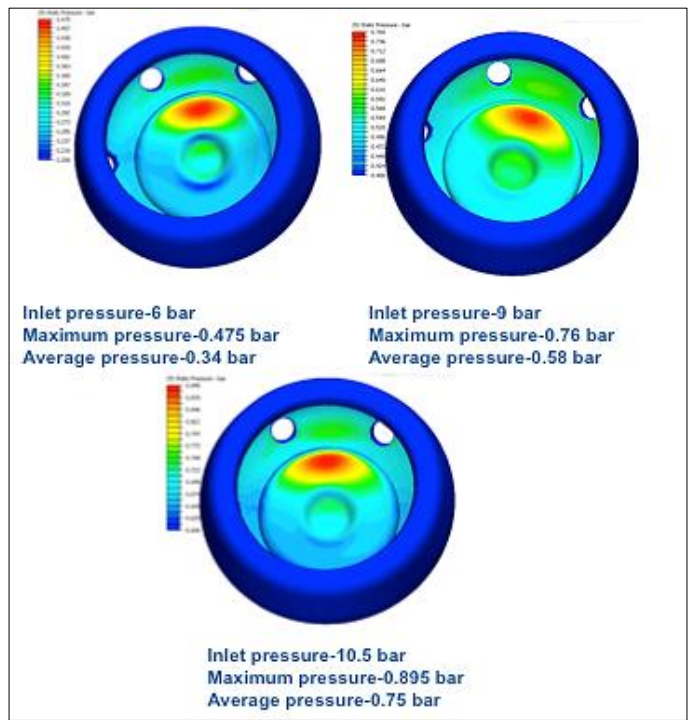

Figure 8-Pressure distribution on protection cover

\section{DESIGN OPTIMIZATION}

Three design iterations were made by modifying the orifice of exhaust protection cover in various combinations and by ensuring flow area of all design iteration is greater than that of existing design as shown in Fig.9.

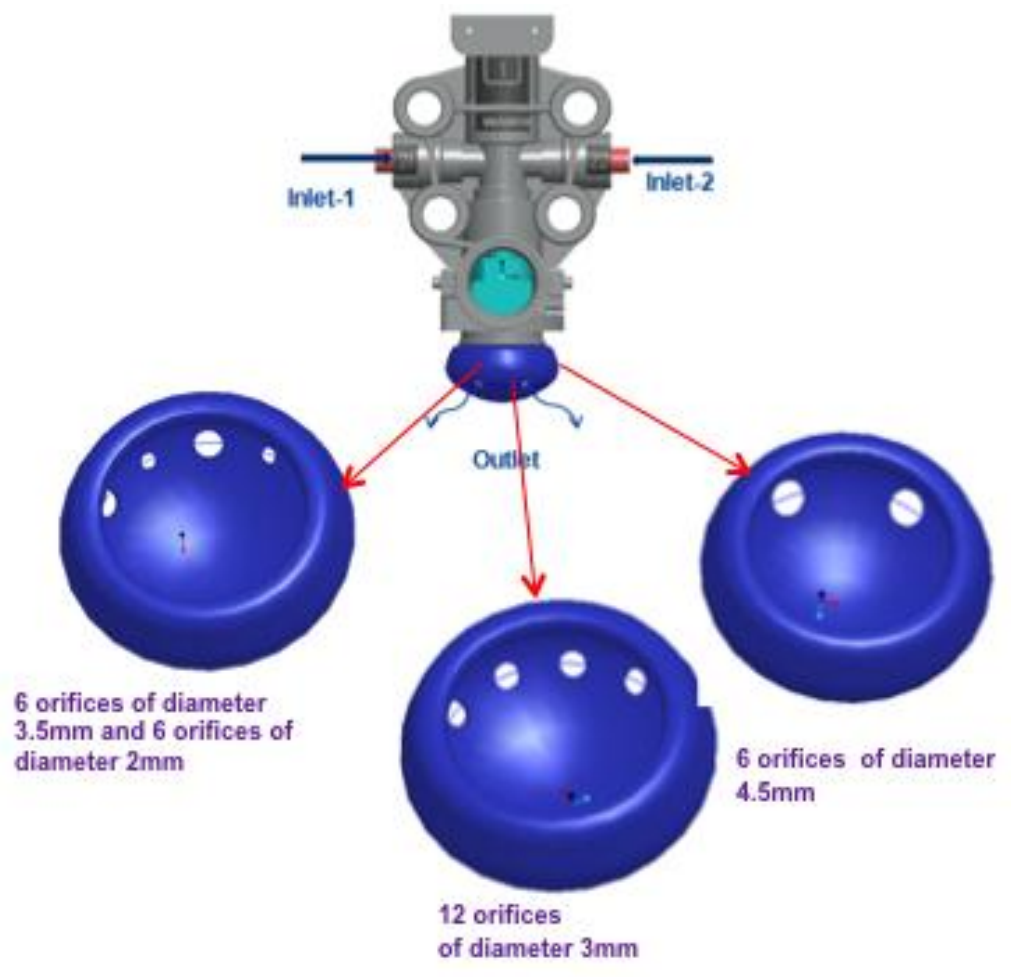

Figure 9-Exhaust protection cover design iteration

\section{Design Iteration 1}

In first iteration with the 6 existing $\varnothing 3.5 \mathrm{~mm}$ orifice 6 additional $\emptyset 2 \mathrm{~mm}$ orifice added and simulated in CFD for pressure distribution on protection cover with inlet pressure of 10.5 bar, pressure distribution plot is shown in Fig.10. The average and maximum pressure distributions are 0.51 bar and 0.68 bar respectively. 


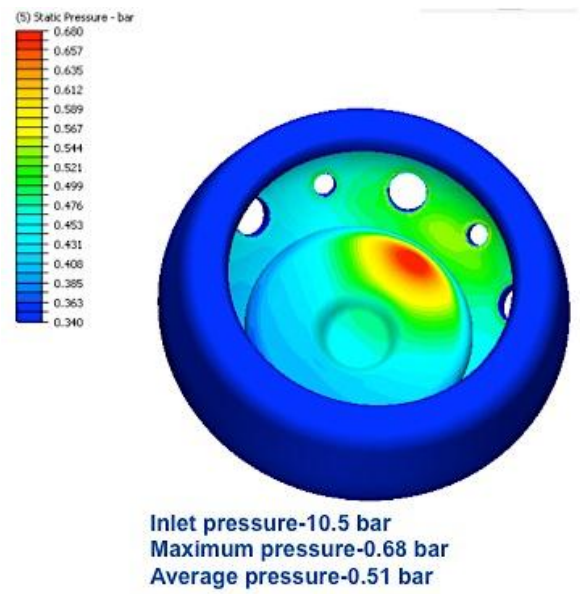

Figure 10-Pressure distribution for iteration-1

\section{Design Iteration 2}

In second iteration 12 numbers of $\varnothing 3 \mathrm{~mm}$ orifice modeled and simulated in CFD for pressure distribution on protection cover with inlet pressure of 10.5 bar, pressure distribution plot is shown in Fig.11. The average and maximum pressure distributions are 0.47 bar and 0.62 bar respectively.

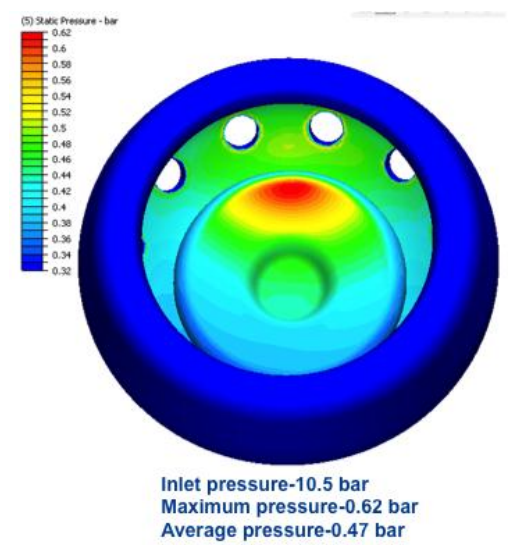

Figure 11-Pressure distribution for iteration-2

\section{Design Iteration 3}

In third iteration 6 numbers of $\varnothing 4.5 \mathrm{~mm}$ orifice modeled and simulated in CFD for pressure distribution on protection cover with inlet pressure of 10.5 bar, pressure distribution plot is shown in Fig.12. The average and maximum pressure distributions are 0.27 bar and 0.335 bar respectively.

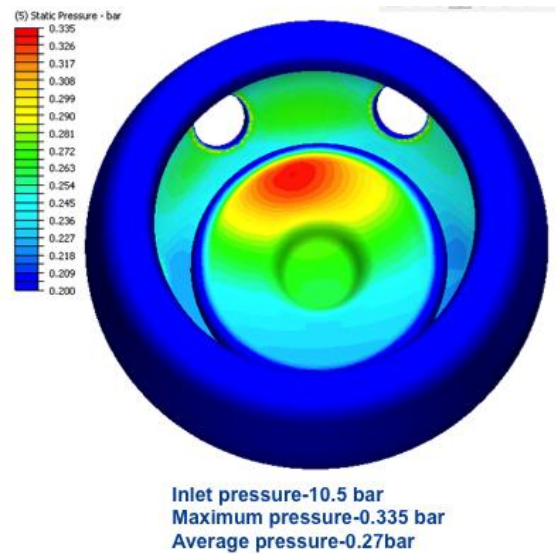

Figure 12-Pressure distribution for iteration-3 


\section{CONCLUSION}

The average and maximum pressure distribution on protection cover for the maximum inlet pressure of 10.5 bar is less than 0.58 bar and 0.76 bar in all three design iterations because of improving the flow area, this means all the three designs will work for maximum pressure requirement even though the flow is high, anyhow design iteration-3 protection cover modeled with 6 orifices of $\varnothing 4.5 \mathrm{~mm}$ is showing lesser pressure than other iterations, also modifying the existing exhaust protection cover for iteration-3 is simple, so it is concluded to go with design iteration-3. The proto sample of the same is made by enlarging the holes of protection cover from $\varnothing 3.5 \mathrm{~mm}$ to $\varnothing 4.5 \mathrm{~mm}$ and tested in same experimental setup. The inlet pressure was increased up to the maximum test bench capacity of 13 bar and the exhaust protection cover passed the test without removal.

Thus for high air flow levelling valve 5 piece exhaust protection cover is replaced with single piece rubber protection cover by optimizing the same using CFD,CFD results and experimental results were comparable and CFD technique is effectively used to optimize the pneumatic flow path and pressure. This analysis reduced the development cycle time and made to understand the physics behind the pneumatics.

\section{Acknowledgements}

I express my gratitude to Dr. Srinivasan, Professor and Director, AU-FRG Institute for CAD/CAM, College of Engineering Guindy, Anna University for allowing me to do the project and continuous encouragement for doing this project work. I also wish to express my deep sense of gratitude to Mr. S. Narayanan, Head of the Department, R\&D, WABCO INDIA Ltd., Chennai, for giving me an opportunity to do the project in the industry research environment.

\section{REFERENCES}

[1] Prabhakar. N., Sripathy. P., Bharathiraja. S., and Ayyappan. K., "Theoretical and Experimental Investigation of Flow Rate of Leveling Valve with Filters for Different Operating Angles," SAE Technical Paper 2013-26-0042.

[2] Suresh Gayakwad. J , Prabhakar. N,Sukumar. T,Shivaraj. V, "Theoretical and Experimental Investigation on back pressure prediction of exhaust brake valve for automotive application," International Conference on Computer Aided Engineering (CAE2013) IIT-Madras, pp.363-370

[3] Moujaes, S. F., and Jagan, R., "3D CFD Predictions and Experimental Comparisons of Pressure Drop in a Ball Valve at Different Partial Openings in Turbulent Flow”. J. Of Energy Engineering, (ASCE_0733-9402), 2008

[4] Autodesk CFD simulation 2012 user's manual 